PROCEEDINGS OF THE

AMERICAN MATHEMATICAL SOCIETY

Volume 129, Number 12, Pages 3559-3567

S 0002-9939(01)06217-7

Article electronically published on May 21, 2001

\title{
HANKEL OPERATORS IN THE BERGMAN SPACE AND SCHATTEN $p$-CLASSES: THE CASE $1<p<2$
}

\author{
JINGBO XIA
}

(Communicated by Joseph A. Ball)

\begin{abstract}
K. Zhu proved in Amer. J. Math. 113 (1991), 147-167, that, for $2 \leq p<\infty$, the Hankel operators $H_{f}$ and $H_{\bar{f}}$ on the Bergman space belong to the Schatten class $\mathcal{C}_{p}$ if and only if the mean oscillation $\operatorname{MO}(f)(z)=$ $\left\{\widetilde{\left.f\right|^{2}}(z)-|\tilde{f}(z)|^{2}\right\}^{1 / 2}$ belongs to $L^{p}\left(D,\left(1-|z|^{2}\right)^{-2} d A(z)\right)$. In this paper we prove that the same result also holds when $1<p<2$.
\end{abstract}

\section{INTRODUCTION}

Let $D$ be the open unit disc $\{z \in \mathbf{C}:|z|<1\}$ in the complex plane and let $d A$ be the area measure on $D$ normalized in such a way that $A(D)=1$. Recall that the Bergman space $L_{a}^{2}(D, d A)$ is defined to be the subspace $\left\{f \in L^{2}(D, d A): f\right.$ is analytic on $D\}$. As is customary, we denote the orthogonal projection from $L^{2}(D, d A)$ to $L_{a}^{2}(D, d A)$ by $P$. Also recall that a Hankel operator $H_{f}: L_{a}^{2}(D, d A) \rightarrow$ $L_{a}^{2}(D, d A)^{\perp}$ with symbol function $f$ is defined by the formula $H_{f}=(1-P) M_{f} P$, where $M_{f}$ is the operator of multiplication by $f$. It is well known that $P$ has $K(z, w)=(1-\bar{w} z)^{-2}$ as its kernel, i.e.,

$$
(P g)(z)=\int K(z, w) g(w) d A(w)=\int \frac{g(w)}{(1-\bar{w} z)^{2}} d A(w) .
$$

Associated with $K$ are the unit vectors $\left\{k_{z}:|z|<1\right\}$ in $L_{a}^{2}(D, d A)$, where

$$
k_{z}(w)=\{K(z, z)\}^{-1 / 2} K(w, z)=\left(1-|z|^{2}\right) /(1-\bar{z} w)^{2} .
$$

Recall that, given an $f \in L^{2}(D, d A)$, its Berezin transform equals

$$
\tilde{f}(z)=\left\langle f k_{z}, k_{z}\right\rangle=\int f\left|k_{z}\right|^{2} d A .
$$

The mean oscillation $\mathrm{MO}(f)$ of $f$ is given by the formula

$$
\operatorname{MO}(f)(z)=\left\{\widetilde{|f|^{2}}(z)-|\tilde{f}(z)|^{2}\right\}^{1 / 2}=\left\{\int|f-\tilde{f}(z)|^{2}\left|k_{z}\right|^{2} d A\right\}^{1 / 2} .
$$

Let $\lambda$ be the measure on $D$ defined by

$$
d \lambda(z)=\left(1-|z|^{2}\right)^{-2} d A(z) .
$$

Received by the editors April 11, 2000.

2000 Mathematics Subject Classification. Primary 47B10, 47B32, 47B35.

This work was supported in part by NSF grant DMS-9703515. 
Recall that, for any $1 \leq p<\infty$, the Schatten $p$-class $\mathcal{C}_{p}$ consists of operators $T$ satisfying the condition $\|T\|_{p}<\infty$, where the $p$-norm is defined by the formula

$$
\|T\|_{p}=\left\{\operatorname{tr}\left(|T|^{p}\right)\right\}^{1 / p}=\left\{\operatorname{tr}\left(\left(T^{*} T\right)^{p / 2}\right)\right\}^{1 / p} .
$$

In the study of Bergman space operators, a persisting theme is to determine the membership of $H_{f}$ in $\mathcal{C}_{p}$. Indeed there is a very rich literature on this subject, of which we cite [1]-4], 6] here as a sample. To explain what we will do in this paper, it is necessary to have a brief review of the relevant history.

In [6], K. Zhu characterized the simultaneous membership $H_{f} \in \mathcal{C}_{p}$ and $H_{\bar{f}} \in \mathcal{C}_{p}$ for the case $2 \leq p<\infty$ and for Hankel operators on the unit ball in $\mathbf{C}^{n}$. In particular, on the unit disc, he proved

Theorem 1 ([6]). Let $2 \leq p<\infty$ and $f \in L^{2}(D, d A)$. Then $H_{f} \in \mathcal{C}_{p}$ and $H_{\bar{f}} \in \mathcal{C}_{p}$ if and only if $M O(f) \in L^{p}(D, d \lambda)$.

Zhu further suggested in [6] that this particular characterization of membership in $\mathcal{C}_{p}$, i.e., the condition $\mathrm{MO}(f) \in L^{p}(D, d \lambda)$, should hold in the case $1<p<2$. This, of course, is precisely what happens if $f$ is assumed to be analytic. Recall that, if $f$ is analytic, then $H_{f}=0$. J. Arazy, S. Fisher and J. Peetre proved the following:

Theorem 2 ([1]). Let $1<p<\infty$ and let $g \in L^{2}(D, d A)$ be an analytic function. Then the following are equivalent:

(a) $H_{\bar{g}} \in \mathcal{C}_{p}$.

(b) $g$ belongs to the Besov space $B_{p}$.

(c) $M O(g) \in L^{p}(D, d \lambda)$.

These authors further showed in [1] that, if $g$ is analytic, then $H_{\bar{g}} \in \mathcal{C}_{1}$ only if $g$ is a constant. This suggests that the membership of Hankel operators in the trace class should perhaps be treated separately. Whether $f$ is analytic or not, the condition $\mathrm{MO}(f) \in L^{1}(D, d \lambda)$ is sufficient, but not necessary, for both $H_{f}$ and $H_{\bar{f}}$ to belong to $\mathcal{C}_{1}$. Indeed, because

$$
\begin{aligned}
\mathrm{MO}(f)(z) & \geq\left\{\int|f-\tilde{f}(z)|^{2} \frac{\left(1-|z|^{2}\right)^{2}}{(1+|z|)^{4}} d A\right\}^{1 / 2} \\
& \geq \frac{1}{4}\left(1-|z|^{2}\right) \inf _{\alpha \in \mathbf{C}}\left\{\int|f-\alpha|^{2} d A\right\}^{1 / 2},
\end{aligned}
$$

the condition $\mathrm{MO}(f) \in L^{1}(D, d \lambda)$ forces $f$ to be a constant on $D$. But obviously there are non-constant functions $f$ on $D$ for which both $H_{f}$ and $H_{\bar{f}}$ are of trace class. For example, if $f$ vanishes outside some $\{z \in \mathbf{C}:|z|<\eta\}, \eta<1$, then both $H_{f}$ and $H_{\bar{f}}$ belong to $\mathcal{C}_{1}$. Nevertheless, Theorem 2 cited above gives one confidence that Theorem 1 should hold true in the case $1<p<2$. In other words, the case $p=1$ should perhaps be viewed as something of an anomaly.

The simultaneous study of both $H_{f}$ and $H_{\bar{f}}$ (or, equivalently, $H_{f}$ for real-valued $f$ ) is usually referred to as a two-sided problem. In his paper [3], among many other results, D. Luecking considered the one-sided problem $H_{f} \in \mathcal{C}_{p}$ for all $1 \leq p<\infty$. In particular we would like to mention the following result, which provides further food for thought.

Theorem 3 ([3]). Let $1 \leq p<2$ and $f \in L^{2}(D, d A)$. Then $H_{f} \in \mathcal{C}_{p}$ if and only if

$$
\int\left\{\int_{D(0)}\left|(1-P)\left(f \circ \varphi_{\zeta}\right)\right|^{2} d A\right\}^{p / 2} d \lambda(\zeta)<\infty,
$$


where $\varphi_{\zeta}(w)=(\zeta-w) /(1-\bar{\zeta} w)$ and $D(0)$ is the disc of some fixed radius $r>0$ in the hyperbolic metric around 0 , i.e., $D(0)=\left\{\zeta \in \mathbf{C}:|\zeta|<\left(e^{r}-1\right) /\left(e^{r}+1\right)\right\}$.

We know that $\operatorname{MO}(f)(z) \leq\left\|(1-P)\left(f \circ \varphi_{z}\right)\right\|+\left\|(1-P)\left(\bar{f} \circ \varphi_{z}\right)\right\|$ (see pages 142 and 143 in 5]). Thus if it were the entire unit disc $D$ that appears in the inner integral in (1.2) instead of $D(0)$, then it would immediately follow that the simultaneous membership $H_{f} \in \mathcal{C}_{p}$ and $H_{\bar{f}} \in \mathcal{C}_{p}$ implies that $\mathrm{MO}(f) \in L^{p}(D, d \lambda)$. But, since Theorem 3 holds true for $p=1$ as well as for $1<p<2$, we know that the $D(0)$ in (1.2) cannot be replaced by $D$ when $p=1$. Then what about the case $1<p<2$ ? Thus we are back to Zhu's original question: Does the simultaneous membership $H_{f} \in \mathcal{C}_{p}$ and $H_{\bar{f}} \in \mathcal{C}_{p}$ imply $\mathrm{MO}(f) \in L^{p}(D, d \lambda)$ when $1<p<2$ ? We feel that, aside from reasons of aesthetics, the extension of Theorem 1 to the case of $1<p<2$ is interesting precisely because it breaks down at $p=1$. Furthermore, because $\mathrm{MO}(f)$ is defined in terms of the Berezin transform, the condition $\mathrm{MO}(f) \in$ $L^{p}(D, d \lambda)$ is of a more global nature than conditions such as (1.2). The purpose of this paper is to show that, when $1<p<2$, the simultaneous membership $H_{f} \in \mathcal{C}_{p}$ and $H_{\bar{f}} \in \mathcal{C}_{p}$ indeed entails $\mathrm{MO}(f) \in L^{p}(D, d \lambda)$ as Zhu originally predicted.

Theorem 4. Let $1<p<2$. Then, for any $f \in L^{2}(D, d A)$, we have $H_{f} \in \mathcal{C}_{p}$ and $H_{\bar{f}} \in \mathcal{C}_{p}$ if and only if $M O(f) \in L^{p}(D, d \lambda)$.

The case $1<p<2$ calls for techniques different from those for the case $2 \leq$ $p<\infty$. For such problems, one would normally consider coverings of $D$ by discs of a fixed radius in the hyperbolic metric. But, to prove Theorem 4, we need to decompose $D$ into circular sectors. This is because the main difficulty in our proof is to use localized mean oscillation to control $\mathrm{MO}(f)$, in which the long-range effect of $\left|k_{z}\right|^{2}$ cannot be ignored. Compared to discs, circular sectors allow us to cover $D$ while avoiding excessive overlapping in an effective way, which is essential for our argument to work. As we will see in the proof of Lemma 7, this system of sectors precisely recaptures the $p=1$ anomaly.

The rest of the paper is organized as follows. In Section 2 we show that the simultaneous membership $H_{f} \in \mathcal{C}_{p}$ and $H_{\bar{f}} \in \mathcal{C}_{p}$ implies the finiteness of two localized mean oscillations associated with $f$, which is not a difficult task. The main part of our work is in Section 3, where we show that these localized mean oscillations can be pieced together to dominate $\mathrm{MO}(f)$.

\section{Decomposition on the DisC}

To prove our main result, we need to use five different kinds of circular sectors in $D$. The reason why so many types of sectors will be used is that we need some of the sectors to overlap, but we must also keep a tight control on overlapping. Fortunately, what mitigates complication is that these five kinds of sectors are really generated by just one kind, $S_{n, j}$, which we will introduce first. For any integers $n \geq 1$ and $1 \leq j \leq 2^{n}$, let

$$
S_{n, j}=\left\{r e^{2 \pi i \theta}: 1-2^{-n}<r<1,2^{-n}(j-1)<\theta \leq 2^{-n} j\right\} .
$$

Such an $S_{n, j}$ is often referred to as a Carleson square. For such $n$ and $j$, we also define

$$
T_{n, j}=\left\{r e^{2 \pi i \theta}: 1-2^{-n}<r \leq 1-2^{-n-1}, 2^{-n}(j-1)<\theta \leq 2^{-n} j\right\} .
$$


That is, $T_{n, j}=S_{n, j} \backslash\left\{\bigcup_{i=1}^{2^{n+1}} S_{n+1, i}\right\}=S_{n, j} \backslash\left\{S_{n+1,2 j-1} \cup S_{n+1,2 j}\right\}$. We further define

$$
\tilde{S}_{n, j}=S_{n, j-1} \cup S_{n, j} \cup S_{n, j+1}, \quad \tilde{T}_{n, j}=T_{n, j-1} \cup T_{n, j} \cup T_{n, j+1}, \quad n \geq 1, \quad 1 \leq j \leq 2^{n},
$$

where we set $S_{n, 0}=S_{n, 2^{n}}, T_{n, 0}=T_{n, 2^{n}}$, and $S_{n, 2^{n}+1}=S_{n, 1}, T_{n, 2^{n}+1}=T_{n, 1}$. Keep in mind that $\tilde{S}_{n, j}$ (resp. $\tilde{T}_{n, j}$ ) is the union of $S_{n, j}$ (resp. $T_{n, j}$ ) with its two immediate neighbors. Finally, for $n \geq 1$ and $1 \leq j \leq 2^{n}$, we define $Q_{n, j}=$ $T_{n, j} \cup T_{n+1,2 j-1} \cup T_{n+1,2 j}$, the union of $T_{n, j}$ with the two $T_{n+1, i}$ 's that are contained in $S_{n, j}$. That is,

$$
Q_{n, j}=\left\{r e^{2 \pi i \theta}: 1-2^{-n}<r \leq 1-2^{-n-2}, 2^{-n}(j-1)<\theta \leq 2^{-n} j\right\} .
$$

For a Borel set $E \subset D$, we will write $|E|$ for $A(E)$. We will also write $\varphi_{E}=$ $\int_{E} \varphi d A /|E|$ when $\varphi \in L^{1}(D, d A)$ and $|E|>0$. As usual, we will denote universal constants by $C_{1}, C_{2}, \ldots$, which may represent different values in the proofs of different lemmas.

Lemma 5. Let $1<p \leq 2$ and let $f \in L^{2}(D, d A)$ be such that $H_{f} \in \mathcal{C}_{p}$ and $H_{\bar{f}} \in \mathcal{C}_{p}$. Then

$$
\sum_{n=1}^{\infty} \sum_{j=1}^{2^{n}}\left(\frac{1}{\left|E_{n, j}\right|} \int_{E_{n, j}}\left|f-f_{E_{n, j}}\right|^{2} d A\right)^{p / 2}<\infty
$$

for $E_{n, j}=\tilde{T}_{n, j}$ and $Q_{n, j}$.

Proof. Define the integral operator $K_{n, j}$ on $L^{2}(D, d A)$ by the formula

$$
\left(K_{n, j} \psi\right)(z)=\chi_{E_{n, j}}(z) \int_{E_{n, j}} \frac{\bar{f}(w)-\bar{f}(z)}{(1-\bar{w} z)^{2}} \psi(w) d A(w) .
$$

Let $q=p /(p-1)$. Since each $K_{n, j}$ is obviously Hilbert-Schmidt and since $q \geq 2$,

$$
\left\|K_{n, j}\right\|_{q}^{2} \leq\left\|K_{n, j}\right\|_{2}^{2}=\int_{E_{n, j}} \int_{E_{n, j}} \frac{|f(w)-f(z)|^{2}}{|1-\bar{w} z|^{4}} d A(w) d A(z) .
$$

Now, $\sup _{z, w \in E_{n, j}}|1-\bar{w} z|^{-2} \leq C_{1}\left|E_{n, j}\right|^{-1}$ by the designs of $\tilde{T}_{n, j}$ and $Q_{n, j}$. Thus

$$
\begin{aligned}
\left\|K_{n, j}\right\|_{q}^{2} & \leq \frac{C_{1}^{2}}{\left|E_{n, j}\right|^{2}} \int_{E_{n, j}} \int_{E_{n, j}}|f(w)-f(z)|^{2} d A(w) d A(z) \\
& =\frac{2 C_{1}^{2}}{\left|E_{n, j}\right|} \int_{E_{n, j}}\left|f-f_{E_{n, j}}\right|^{2} d A .
\end{aligned}
$$

Define $\alpha_{n, j}=\left\{\int_{E_{n, j}}\left|f-f_{E_{n, j}}\right|^{2} d A /\left|E_{n, j}\right|\right\}^{(p-2) / 2}$ if $\int_{E_{n, j}}\left|f-f_{E_{n, j}}\right|^{2} d A>0$ and define $\alpha_{n, j}=0$ if that integral equals 0 .

Define $K_{N}=\sum_{n=1}^{N} \sum_{j=1}^{2^{n}} \alpha_{n, j} K_{n, j}$ for any integer $N \geq 1$. We next show that

$$
\left\|K_{N}\right\|_{q} \leq C_{2}\left\{\sum_{n=1}^{N} \sum_{j=1}^{2^{n}}\left(\frac{1}{\left|E_{n, j}\right|} \int_{E_{n, j}}\left|f-f_{E_{n, j}}\right|^{2} d A\right)^{p / 2}\right\}^{1 / q} .
$$

Let us first consider the case where $E_{n, j}=\tilde{T}_{n, j}$. In this case we write

$$
K_{N}=A_{0}+A_{1}+A_{2}, \quad \text { where } A_{i}=\sum_{n=1}^{N} \sum_{j=i \bmod 3} \alpha_{n, j} K_{n, j}, \quad i=0,1,2 .
$$


Since $\tilde{T}_{n, j}=T_{n, j-1} \cup T_{n, j} \cup T_{n, j+1}$, for each $i \in\{0,1,2\}$, the subspaces $\left\{L^{2}\left(\tilde{T}_{n, j}, d A\right)\right.$ : $\left.1 \leq n \leq N, 1 \leq j \leq 2^{n}, j=i \bmod 3\right\}$ are pairwise orthogonal. Therefore

$$
\left\|A_{i}\right\|_{q}=\left\{\sum_{n=1}^{N} \sum_{j=i \bmod 3} \alpha_{n, j}^{q}\left\|K_{n, j}\right\|_{q}^{q}\right\}^{1 / q} .
$$

By (2.2) and the definition of $\alpha_{n, j}$, we have

$$
\alpha_{n, j}^{q}\left\|K_{n, j}\right\|_{q}^{q} \leq\left(2 C_{1}^{2}\right)^{q / 2}\left\{\frac{1}{\left|E_{n, j}\right|} \int_{E_{n, j}}\left|f-f_{E_{n, j}}\right|^{2} d A\right\}^{(q(p-2) / 2)+(q / 2)} .
$$

Since $(q(p-2) / 2)+(q / 2)=p / 2,(2.3)$ follows from $(2.4-2.6)$ when $E_{n, j}=\tilde{T}_{n, j}$.

In the case where $E_{n, j}=Q_{n, j}$, we decompose $K_{N}$ as $K_{N}=B_{0}+B_{1}$ with

$$
B_{i}=\sum_{\substack{1 \leq n \leq N \\ n=i \bmod 2}} \sum_{j=1}^{2^{n}} \alpha_{n, j} K_{n, j}, \quad i=0,1 .
$$

Again, for each $i \in\{0,1\}$, the subspaces $\left\{L^{2}\left(Q_{n, j}, d A\right): 1 \leq n \leq N, n=i \bmod 2\right.$, $\left.1 \leq j \leq 2^{n}\right\}$ are pairwise orthogonal. Thus the rest of the proof of (2.3) in the case $E_{n, j}=Q_{n, j}$ is the same as in the previous case. This completes the proof of (2.3).

Note that $\left[M_{f}, P\right]=\left[M_{f}, P\right] P+\left[M_{f}, P\right](1-P)=H_{f}-P M_{f}(1-P)=H_{f}-$ $\left(H_{\bar{f}}\right)^{*} \in \mathcal{C}_{p}$ under the assumption of the lemma. It is easy to see that

$$
\operatorname{tr}\left(\left[M_{f}, P\right] K_{N}\right)=\sum_{n=1}^{N} \sum_{j=1}^{2^{n}} \alpha_{n, j} \int_{E_{n, j}} \int_{E_{n, j}} \frac{|f(w)-f(z)|^{2}}{|1-\bar{w} z|^{4}} d A(w) d A(z) .
$$

Now $\inf _{z, w \in E_{n, j}}|1-\bar{w} z|^{-2} \geq c_{3}\left|E_{n, j}\right|^{-1}$. Since $\left\|\left[M_{f}, P\right]\right\|_{p}\left\|K_{N}\right\|_{q} \geq \operatorname{tr}\left(\left[M_{f}, P\right] K_{N}\right)$, it follows from (2.7) that

$$
\begin{aligned}
\left\|\left[M_{f}, P\right]\right\|_{p}\left\|K_{N}\right\|_{q} & \geq \sum_{n=1}^{N} \sum_{j=1}^{2^{n}} \alpha_{n, j} \frac{c_{3}^{2}}{\left|E_{n, j}\right|^{2}} \int_{E_{n, j}} \int_{E_{n, j}}|f(w)-f(z)|^{2} d A(w) d A(z) \\
& =2 c_{3}^{2} \sum_{n=1}^{N} \sum_{j=1}^{2^{n}}\left(\frac{1}{\left|E_{n, j}\right|^{2}} \int_{E_{n, j}}\left|f-f_{E_{n, j}}\right|^{2} d A\right)^{p / 2} .
\end{aligned}
$$

It is easy to see that (2.1) follows from this inequality and (2.3).

Note that Lemma 5 also holds in the case $p=1$. In this case, instead of $(2.3)$, it is easy to derive the operator-norm bound $\sup _{N}\left\|K_{N}\right\|<\infty$. Thus from the trace estimate above one also obtains (2.1) when $p=1$.

\section{Assembling the pieces}

The proof of Lemma 5 was easy because, in the case $E_{n, j}=\tilde{T}_{n, j}$ or $Q_{n, j}$, the diameter of $E_{n, j}$ is comparable to its distance from the boundary $\partial D$ of the disc. Real work comes when we deal with $S_{n, j}$ and $\tilde{S}_{n, j}$, which extend all the way to $\partial D$. To reduce the amount of writing, in the sequel we will use the notation

$$
V(f ; E)=\frac{1}{|E|} \int_{E}\left|f-f_{E}\right|^{2} d A .
$$


Lemma 6. Let $1 \leq p \leq 2$ and let $f \in L^{2}(D, d A)$ be such that

$$
\sum_{n=1}^{\infty} \sum_{j=1}^{2^{n}}\left(\frac{1}{\left|E_{n, j}\right|} \int_{E_{n, j}}\left|f-f_{E_{n, j}}\right|^{2} d A\right)^{p / 2}<\infty
$$

for $E_{n, j}=\tilde{T}_{n, j}$ and $Q_{n, j}$. Then (3.1) also holds when $E_{n, j}=\tilde{S}_{n, j}$.

Proof. We claim that, to prove the lemma, it suffices to establish

$$
\sum_{n=1}^{\infty} \sum_{j=1}^{2^{n}}\left(\frac{1}{\left|S_{n, j}\right|} \int_{S_{n, j}}\left|f-f_{T_{n, j}}\right|^{2} d A\right)^{p / 2}<\infty
$$

Indeed, recalling the definition that $\tilde{S}_{n, j}=S_{n, j-1} \cup S_{n, j} \cup S_{n, j+1}$, we have

$$
\begin{aligned}
V\left(f ; \tilde{S}_{n, j}\right) & \leq \frac{1}{\left|\tilde{S}_{n, j}\right|} \int_{\tilde{S}_{n, j}}\left|f-f_{\tilde{T}_{n, j}}\right|^{2} d A=\frac{1}{3} \sum_{i=-1}^{1} \frac{1}{\left|S_{n, j+i}\right|} \int_{S_{n, j+i}}\left|f-f_{\tilde{T}_{n, j}}\right|^{2} d A \\
& \leq \frac{1}{3} \sum_{i=-1}^{1} \frac{1}{\left|S_{n, j+i}\right|} \int_{S_{n, j+i}} 2\left\{\left|f-f_{T_{n, j+i}}\right|^{2}+\left|f_{T_{n, j+i}}-f_{\tilde{T}_{n, j}}\right|^{2}\right\} d A \\
& \leq \frac{2}{3} \sum_{i=-1}^{1} \frac{1}{\left|S_{n, j+i}\right|} \int_{S_{n, j+i}}\left|f-f_{T_{n, j+i}}\right|^{2} d A+6 V\left(f ; \tilde{T}_{n, j}\right),
\end{aligned}
$$

where the third $\leq$ is due to the fact that $\left|f_{E}-f_{F}\right|^{2} \leq \int_{E}\left|f-f_{F}\right|^{2} d A /|E| \leq$ $(|F| /|E|) V(f ; F)$ when $E \subset F$. Hence the conclusion of the lemma follows from (3.2).

To prove (3.2), we fix a pair of $n, j$ and write $S=S_{n, j}, T=T_{n, j}$ for the moment. Since $S=\bigcup\left\{T_{k, s}: k \geq n, T_{k, s} \subset S\right\}$ and $\left|T_{k, s}\right| /|S| \leq C_{1} 2^{-2(k-n)}$ when $k \geq n$, we have

$$
\begin{aligned}
\frac{1}{|S|} \int_{S}\left|f-f_{T}\right|^{2} d A & \leq C_{1} \sum_{T_{k, s} \subset S} 2^{-2(k-n)} \frac{1}{\left|T_{k, s}\right|} \int_{T_{k, s}}\left|f-f_{T}\right|^{2} d A \\
& \leq 2 C_{1} \sum_{T_{k, s} \subset S} 2^{-2(k-n)}\left(V\left(f ; T_{k, s}\right)+\left|f_{T_{k, s}}-f_{T}\right|^{2}\right) .
\end{aligned}
$$

Given a $T_{k, s} \subset S$ with $k>n$, there is a unique chain of pairs of indices $(n, j)=$ $(n, s(0)),(n+1, s(1)), \ldots,(k-1, s(k-n-1)),(k, s(k-n))=(k, s)$ such that $T_{n+t, s(t)} \cup T_{n+t+1, s(t+1)} \subset Q_{n+t, s(t)}$ for $t=0, \ldots, k-n-1$. Thus

$$
\left|f_{T}-f_{T_{k, s}}\right| \leq \sum_{t=0}^{k-n-1}\left|f_{T_{n+t, s(t)}}-f_{T_{n+t+1, s(t+1)}}\right| \leq C_{2} \sum_{t=0}^{k-n-1}\left\{V\left(f ; Q_{n+t, s(t)}\right)\right\}^{1 / 2},
$$

where we used the fact that $\left\{V\left(f ; Q_{n+t, s(t)}\right)\right\}^{1 / 2}$ dominates both $\mid f_{T_{n+t, s(t)}}-$ $f_{Q_{n+t, s(t)}} \mid$ and $\left|f_{T_{n+t+1, s(t+1)}}-f_{Q_{n+t, s(t)} \mid}\right|$. It is also easy to see that $V\left(f ; T_{k, s}\right) \leq$ $C_{3} V\left(f ; Q_{k, s}\right)$. Applying the Cauchy-Schwarz inequality to (3.4), we have

$$
V\left(f ; T_{k, s}\right)+\left|f_{T}-f_{T_{k, s}}\right|^{2} \leq C_{4}\left\{V\left(f ; Q_{k, s}\right)+(k-n) \sum_{t=0}^{k-n-1} V\left(f ; Q_{n+t, s(t)}\right)\right\} .
$$

Note that the chain $(n, j)=(n, s(0)),(n+1, s(1)), \ldots,(k-1, s(k-n-1))$, $(k, s(k-n))=(k, s)$ consists precisely of those pairs of indices $(\nu, i)$ such that 
the relation $T_{k, s} \subset S_{\nu, i} \subset S$ holds. Since the above applies to every $T_{k, s} \subset S$, substituting (3.5) into (3.3) and observing that $k-n \leq C_{5} 2^{(k-n) / 2}$, we obtain

$$
\frac{1}{|S|} \int_{S}\left|f-f_{T}\right|^{2} d A \leq C_{6} \sum_{T_{k, s} \subset S_{\nu, i} \subset S} 2^{-3(k-n) / 2} V\left(f ; Q_{\nu, i}\right) .
$$

Now, for each $S_{\nu, i} \subset S$ and for each $k \geq \nu$, the cardinality of the set $\left\{s: 1 \leq s \leq 2^{k}\right.$, $\left.T_{k, s} \subset S_{\nu, i}\right\}$ equals $2^{k-\nu}$. Thus a rearrangement of the terms in (3.6) gives us

$$
\begin{aligned}
& \frac{1}{|S|} \int_{S}\left|f-f_{T}\right|^{2} d A \\
& \quad \leq C_{6} \sum_{S_{\nu, i} \subset S} V\left(f ; Q_{\nu, i}\right) \sum_{k=\nu}^{\infty} 2^{-3(k-n) / 2} \operatorname{card}\left\{s: T_{k, s} \subset S_{\nu, i}\right\} \\
& \quad=C_{6} \sum_{S_{\nu, i} \subset S} V\left(f ; Q_{\nu, i}\right) \sum_{k=\nu}^{\infty} 2^{-(k-n) / 2} \cdot 2^{-k+n} \cdot 2^{k-\nu} \\
& \quad \leq C_{7} \sum_{S_{\nu, i} \subset S} 2^{-\nu+n} V\left(f ; Q_{\nu, i}\right) .
\end{aligned}
$$

Note that $\left(\sum a_{\nu}\right)^{r} \leq \sum a_{\nu}^{r}$ if $0<r \leq 1$ and $a_{\nu} \geq 0$. Since $p / 2 \leq 1$, (3.7) implies

$$
\left\{\frac{1}{|S|} \int_{S}\left|f-f_{T}\right|^{2} d A\right\}^{p / 2} \leq C_{8} \sum_{S_{\nu, i} \subset S}\left\{2^{-\nu+n} V\left(f ; Q_{\nu, i}\right)\right\}^{p / 2} .
$$

This, of course, holds true for any given $S=S_{n, j}$ and $T=T_{n, j}$. Summing over all possible indices $n$ and $j$, from (3.8) we obtain

$$
\begin{aligned}
\sum_{n, j}\left(\frac{1}{\left|S_{n, j}\right|} \int_{S_{n, j}}\left|f-f_{T_{n, j}}\right|^{2} d A\right)^{p / 2} & \leq C_{8} \sum_{n, j} \sum_{S_{\nu, i} \subset S_{n, j}} 2^{-p(\nu-n) / 2}\left(V\left(f ; Q_{\nu, i}\right)\right)^{p / 2} \\
& =C_{8} \sum_{\nu, i}\left(V\left(f ; Q_{\nu, i}\right)\right)^{p / 2} \sum_{S_{n, j} \supset S_{\nu, i}} 2^{-p(\nu-n) / 2} .
\end{aligned}
$$

Observe that, for each given pair of $\nu, i$ and each $n \leq \nu$, there is precisely one $j$ such that $S_{n, j} \supset S_{\nu, i}$. That is, $\sum_{S_{n, j} \supset S_{\nu, i}} 2^{-p(\nu-n) / 2}=\sum_{n=1}^{\nu} 2^{-p(\nu-n) / 2}$ for any $\nu$, $i$. Since the lemma assumes (3.1) for $E_{n, j}=Q_{n, j}$, the above is finite. This proves (3.2).

What we have done so far works in the case $p=1$ as well as in the case $1<p \leq 2$. Our next lemma is the one that cannot be extended to the case $p=1$.

Lemma 7. Suppose that $1<p \leq 2$. Let $f \in L^{2}(D, d A)$ be such that (3.1) holds for $E_{n, j}=\tilde{S}_{n, j}$. Then $M O(f) \in L^{p}(D, d \lambda)$, where $d \lambda(z)=\left(1-|z|^{2}\right)^{-2} d A(z)$.

Proof. From the definition of the sectors $T_{n, j}$ it is easy to deduce that $\sup _{n, j} \lambda\left(T_{n, j}\right)$ $<\infty$. Since $\bigcup_{n, j} T_{n, j}=\{z: 1 / 2<|z|<1\}$, to prove the lemma, it suffices to establish

$$
\sum_{n, j} \sup _{z \in T_{n, j}}\{\operatorname{MO}(f)(z)\}^{p}<\infty .
$$

Again, it follows from the definition of $T_{n, j}$ that, for any $z \in T_{n, j}$,

$$
\sup _{w \in D}\left|k_{z}(w)\right|^{2} \leq \frac{\left(1-|z|^{2}\right)^{2}}{(1-|z|)^{4}} \leq C_{1}\left(2^{-2 n} / 2^{-4 n}\right) \leq C_{2}\left|\tilde{S}_{n, j}\right|^{-1}
$$


By the definition of $\tilde{S}_{\ell, t}$, if $\ell<n, S_{\ell, t} \supset S_{\ell+1, \tau} \supset S_{n, j}$ and $z \in T_{n, j}$, then

$$
\sup _{w \in D \backslash \tilde{S}_{\ell+1, \tau}}\left|k_{z}(w)\right|^{2} \leq C_{3}\left(2^{-2 n} / 2^{-4(\ell+1)}\right) \leq C_{4} 2^{-2(n-\ell)}\left|\tilde{S}_{\ell, t}\right|^{-1} .
$$

Thus, if we set $S_{0,1}=\tilde{S}_{0,1}=D$, then for any $g \in L^{1}(D, d A)$ and any $n, j$, we have

$$
\sup _{z \in T_{n, j}} \int|g|\left|k_{z}\right|^{2} d A \leq C_{5} \sum_{S_{\ell, t} \supset S_{n, j}} 2^{-2(n-\ell)} \frac{1}{\left|\tilde{S}_{\ell, t}\right|} \int_{\tilde{S}_{\ell, t}}|g| d A .
$$

Fix a pair of $n, j$ for the moment and let $(0,1)=(0, t(0)),(1, t(1)), \ldots$, $(n-1, t(n-1)),(n, t(n))=(n, j)$ be the pairs of indices such that $S_{\ell, t(\ell)} \supset S_{n, j}$ for all $0 \leq \ell \leq n$. Let us also write $S_{\ell}=S_{\ell, t(\ell)}$ and $\tilde{S}_{\ell}=\tilde{S}_{\ell, t(\ell)}, 0 \leq \ell \leq n$, for the moment. If $\ell<n$, then

$$
\begin{aligned}
\frac{1}{\left|\tilde{S}_{\ell}\right|} \int_{\tilde{S}_{\ell}}\left|f-f_{\tilde{S}_{n}}\right|^{2} d A & \leq 2\left\{V\left(f ; \tilde{S}_{\ell}\right)+\left|f_{\tilde{S}_{\ell}}-f_{\tilde{S}_{n}}\right|^{2}\right\} \\
& \leq 2\left\{V\left(f ; \tilde{S}_{\ell}\right)+(n-\ell) \sum_{\nu=\ell}^{n-1}\left|f_{\tilde{S}_{\nu}}-f_{\tilde{S}_{\nu+1}}\right|^{2}\right\} \\
& \leq 2(n-\ell+1) \sum_{\nu=\ell}^{n-1} 4 V\left(f ; \tilde{S}_{\nu}\right),
\end{aligned}
$$

where, as we recall, $V(f ; E)=\int\left|f-f_{E}\right|^{2} d A /|E|$. By (3.10) and (3.11), for any $z \in T_{n, j}$,

$$
\begin{aligned}
\{\operatorname{MO}(f)(z)\}^{2} & \leq \int\left|f-f_{\tilde{S}_{n, j}}\right|^{2}\left|k_{z}\right|^{2} d A \\
& \leq C_{5} \sum_{\ell=0}^{n} 2^{-2(n-\ell)} \frac{1}{\left|\tilde{S}_{\ell}\right|} \int_{\tilde{S}_{\ell}}\left|f-f_{\tilde{S}_{n}}\right|^{2} d A \\
& \leq C_{6} \sum_{\ell=0}^{n} 2^{-2(n-\ell)}(n-\ell+1) \sum_{\nu=\ell}^{n} V\left(f ; \tilde{S}_{\nu}\right) \\
& =C_{6} \sum_{\nu=0}^{n} V\left(f ; \tilde{S}_{\nu}\right) \sum_{\ell=0}^{\nu} 2^{-2(n-\ell)}(n-\ell+1) \\
& \leq C_{6} \sum_{\nu=0}^{n} V\left(f ; \tilde{S}_{\nu}\right) \sum_{k=n-\nu}^{\infty} 2^{-2 k}(k+1) .
\end{aligned}
$$

We now use the condition $p>1$ : Since $p / 2>1 / 2$, there is an $\epsilon>0$ such that $(p / 2)(2-\epsilon)>1$. Thus if we set $C_{7}=C_{6} \sum_{k=0}^{\infty} 2^{-\epsilon k}(k+1)$, then from (3.12) we obtain

$$
\begin{aligned}
\sup _{z \in T_{n, j}}\{\operatorname{MO}(f)(z)\}^{2} & \leq C_{7} \sum_{\nu=0}^{n} 2^{-(2-\epsilon)(n-\nu)} V\left(f ; \tilde{S}_{\nu}\right) \\
& =C_{7} \sum_{S_{\ell, t} \supset S_{n, j}} 2^{-(2-\epsilon)(n-\ell)} V\left(f ; \tilde{S}_{\ell, t}\right)
\end{aligned}
$$

upon recalling the notation $\tilde{S}_{\ell}=\tilde{S}_{\ell, t(\ell)}$ with $S_{\ell, t(\ell)} \supset S_{n, j}$ used above. 
Since $p / 2 \leq 1$, we have $\left(\sum a_{\ell}\right)^{p / 2} \leq \sum a_{\ell}^{p / 2}$ if $a_{\ell} \geq 0$. Therefore (3.13) implies

$$
\sup _{z \in T_{n, j}}\{\mathrm{MO}(f)(z)\}^{p} \leq C_{8} \sum_{S_{\ell, t} \supset S_{n, j}} 2^{-\beta(n-\ell)}\left(V\left(f ; \tilde{S}_{\ell, t}\right)\right)^{p / 2},
$$

where $\beta=(2-\epsilon)(p / 2)$. Now, given a pair of $\ell, t$ and given any $n \geq \ell$, there are exactly $2^{n-\ell}$ sectors $S_{n, j}$ contained in $S_{\ell, t}$. Hence

$$
\begin{aligned}
\sum_{n, j} \sup _{z \in T_{n, j}}\{\mathrm{MO}(f)(z)\}^{p} & \leq C_{8} \sum_{n, j} \sum_{S_{\ell, t} \supset S_{n, j}} 2^{-\beta(n-\ell)}\left(V\left(f ; \tilde{S}_{\ell, t}\right)\right)^{p / 2} \\
& =C_{8} \sum_{\ell, t}\left(V\left(f ; \tilde{S}_{\ell, t}\right)\right)^{p / 2} \sum_{n \geq \ell} 2^{-\beta(n-\ell)} \operatorname{card}\left\{j: S_{n, j} \subset S_{\ell, t}\right\} \\
& =C_{8} \sum_{\ell, t}\left(V\left(f ; \tilde{S}_{\ell, t}\right)\right)^{p / 2} \sum_{n \geq \ell} 2^{-(\beta-1)(n-\ell)} .
\end{aligned}
$$

Since $\beta=(2-\epsilon)(p / 2)>1$ by the choice of $\epsilon$, this quantity is finite whenever (3.1) holds for $E_{n, j}=\tilde{S}_{n, j}$. This proves (3.9) and completes the proof of the lemma.

Proof of Theorem 4. Let $1<p<2$ and $f \in L^{2}(D, d A)$ as in the statement of the theorem. It follows from Lemmas 5,6 and 7 that the simultaneous membership $H_{f} \in \mathcal{C}_{p}$ and $H_{\bar{f}} \in \mathcal{C}_{p}$ implies $\mathrm{MO}(f) \in L^{p}(D, d A)$. The converse of this follows from a well-known argument, which we repeat below in the interest of completeness.

By an argument due to Zhu [4], $\left\|H_{f} k_{z}\right\|^{2}=\left\|f k_{z}\right\|^{2}-\left\|P f k_{z}\right\|^{2} \leq\left\|f k_{z}\right\|^{2}-$ $\left|\left\langle P f k_{z}, k_{z}\right\rangle\right|^{2}=\{\mathrm{MO}(f)(z)\}^{2}$. Thus if $\mathrm{MO}(f)$ belongs to $L^{p}(D, d \lambda)$, then so does the function $z \mapsto\left\|H_{f} k_{z}\right\|$. Since $p / 2 \leq 1$, we have

$$
\left\langle\left(H_{f}^{*} H_{f}\right)^{p / 2} k_{z}, k_{z}\right\rangle \leq\left\langle H_{f}^{*} H_{f} k_{z}, k_{z}\right\rangle^{p / 2}=\left\|H_{f} k_{z}\right\|^{p}
$$

and

$$
\operatorname{tr}\left(\left(H_{f}^{*} H_{f}\right)^{p / 2}\right)=\int\left\langle\left(H_{f}^{*} H_{f}\right)^{p / 2} k_{z}, k_{z}\right\rangle d \lambda(z) \leq \int\left\|H_{f} k_{z}\right\|^{p} d \lambda(z)
$$

(see pages 115-117 in [5]). Hence $H_{f} \in \mathcal{C}_{p}$ when $\operatorname{MO}(f) \in L^{p}(D, d \lambda)$. Since $\operatorname{MO}(\bar{f})$ $=\mathrm{MO}(f)$, we also obtain $H_{\bar{f}} \in \mathcal{C}_{p}$ under the condition $\mathrm{MO}(f) \in L^{p}(D, d \lambda)$.

\section{REFERENCES}

1. J. Arazy, S. Fisher and J. Peetre, Hankel operators on weighted Bergman spaces, Amer. J. Math. 110 (1988), 989-1054. MR 90a:47067

2. H. Li and D. Luecking, Schatten class of Hankel operators and Toeplitz operators on the Bergman space of strongly pseudoconvex domains, Contemp. Math. 185 (Multivariable Operator Theory, R. Curto et al., eds., 1995), 237-257. MR 96c:47038

3. D. Luecking, Characterization of certain classes of Hankel operators on the Bergman spaces of the unit disc, J. Funct. Anal. 110 (1992), 247-271. MR 93j:47039

4. K. Zhu, Hilbert-Schmidt Hankel operators on the Bergman space, Proc. Amer. Math. Soc. 109 (1990), 721-730. MR 90k:47060

5. K. Zhu, Operator theory in function spaces, Marcel and Dekker, New York, 1990. MR 92c:47031

6. K. Zhu, Schatten class Hankel operators on the Bergman space of the unit ball, Amer. J. Math. 113 (1991), 147-167. MR 91m:47036

Department of Mathematics, State University of New York at Buffalo, Buffalo, NEW YORK 14260

E-mail address: jxia@acsu.buffalo.edu 